\title{
Taxonomy for GP-Aware Mobility
}

\author{
Sérgio Figueiredo ${ }^{1}$, Justino Lourenço ${ }^{1,2,3}$, Rui Aguiar ${ }^{1,4}$, and Augusto Neto ${ }^{5}$ \\ ${ }^{1}$ Instituto de Telecomunicações de Aveiro, 3810-193 Aveiro, Portugal \\ ${ }^{2}$ Inst. Superior Politécnico Gaya, 4400-103 Santa Marinha - Vila Nova de Gaia, Portugal \\ ${ }^{3}$ Universidade de Vigo, C.P. 36310 Vigo, Spain \\ ${ }^{4}$ Universidade de Aveiro, 3810-193 Aveiro, Portugal \\ ${ }^{5}$ Instituto de Informática, Universidade Federal de Goiás, Brazil \\ \{sfigueiredo,jml,ruilaa) @av.it.pt, augusto@inf.ufg.br
}

\begin{abstract}
We present a structured analysis for classification of diverse mobility schemes, resulting in a taxonomy for mobility in Future Internet systems. The different approaches discussed are based on the Generic Path (GP) concept, a unified framework for the transport of information, and all of them revolve around the existence of a binding between the user and the end-to-end path. Each of the schemes is mappable to real existing and envisioned scenarios, and cover a broad type of services, such as conversational, streaming or interactive ones. As a base to this structured analysis, the work introduces the concept of Generic Path Management Record (GPMR), a flexible record capable of storing relevant information for any type of path, at any level, such as throughput, delay SNR or even authentication paramenters. Thereby, GPMR behaves as much more than a mobility tool, extending its usefulness to everything related to the Network Management universe.
\end{abstract}

Keywords: 4WARD[1], Future Internet, GP, GPMR, Mobility support.

\section{Introduction}

The evolution of the actual Internet is a major current concern in the Telecommunications field, with the all-mighty Internet model, supported by the TCP/IP stack, showing its age. This model was created considering simple information transfer, such as file transfer and messages, in-between a restricted number of trusted nodes interconnected by copper. Such simplistic vision became increasingly inadequate to evolving user needs, and some alternative patches to this became commonly discussed, such as IPv6 [2] MIPv6 [3] DCCP [4]or SCTP [5] Part of the issues raised by the integration of new protocols in the Internet are correlated with the experience of more and more complex scenarios, mainly due to heterogeneity. For instance, it is noted the increasing demand for video streaming sessions (e.g., IPTV) by group of users simultaneously. In such scenario, users (mobile or not and supporting different network interfaces) of the same group can be connected to different network technologies (WiFi, WiMAX, 3G, etc.), as well as receive the data content through different transport schemes (broadcast, unicast and/or multicast). Thus, the simple Internet initial model has deeply changed in order to support set of new functionalities. Additionally, the 
end-to-end model has been questioned long ago: a simple HTTP session, originally intended for as an end-to-end transaction, is now frequently intercepted by firewalls and proxies along the path. As a matter of fact, in spite of the huge success of the current Internet architecture, it is clear that we are not facing the ideal solution since it does not fit the needs brought by such new elements as optical communications systems or the inclusion of mobility and security as guaranteed features. A new Internet framework should take into account aspects and questions like naming, addressing, routing, QoS, self-management, seamless mobility, reliability and availability, interoperability, scalability, security, power consumption optimization and performance enhancement. Heavy investment was put for researching the proper evolution for the Internet ([6][6], [7][7], [8][8]), with proposals alternating between the introduction of single-problem solutions, and the design of a clean slate architecture, both taking into consideration the foreseen services and characteristics that Future Internet should supply.

This work focuses in the ways mobility can be assured in future models. The attempt to add efficient mobility to the current Internet architecture is handicapped, in a great part due to the locator-identifier dependence, with current solutions for Internet naming based on the host address, typically the IP address. In order to provide routing scalability, the actual model uses a hierarchical method of addressing, which implies that the host address has an intrinsic relation with its location. Most works regarding this issue start out by breaking out the hierarchical routing scheme or by rebuilding the IP addressing, which in other words means the redefinition of the Internet structure itself. One of these approaches is the IST 7th Framework Programme Integrated Project 4WARD, which aims to combine the innovations needed to improve the operation of any single network architecture with the coexistence / interoperability of diverse and complementary network architectures in a unique framework. Inside 4WARD, the concept of GP [9] has been introduced [9], [10], which brings an abstract way of describing the transport of information between (two or more) communicating parties, whatever the type of communication is, and improving operational efficiency for both the user and network. As we discuss in Section 2, in order to support this framework, a new functional element is introduced, the GPMR, a record designed for storing all relevant information of the GP, such as QoS parameters and associated End Points (EPs). Taking into account that the GP can be defined at any communication level, the information that GPMR will store is distinct according to the corresponding GP level. This is a major characteristic that shows the relevance and usefulness, in particular for mobility support. In Section 3[11], we present a four elements taxonomy for mobility within GP architecture [11], and conclude the paper in Section 4.

\section{The Generic Path Concept}

Networks share a content delivery task between sources and destination nodes that may be linked by different types of physical technologies, with information traveling by traversing relay nodes that simply provide sub-paths. These sub-paths, when compounded, form the end-to-end path, built with the support of several layers. 
Based on such reality, 4WARD approach brings the concept of Generic Path (GP) as an abstract form of information transport that can either represent a physical pointto-multipoint connection or the distributed information exchange between applications. For this to be feasible, the GP concept follows an object oriented approach, allowing its instantiation to benefit from class specific characteristics, well adapted to the aimed transport service. By definition, GPs start and end at EPs. A GP can have more than one source of information connected to more than one sink of information, in a multi-point to multi-point way, this way redefining the concept of end-to-end.

\subsection{Generic Path Architecture Elements}

In the proposed 4WARD Generic Path architecture several blocks were introduced and will be briefly described in this section.

A Generic Path is a means for transferring information between two or more End Points. An EP acts as the interface between the GP termination and the Entity (i.e., the client), and it uses an identifier, the EID (Endpoint Identifier). The bootstrap of a new GP is done by a functional component called GP Factory, which exists within each compartment (CT, described later).

Concerning the morphology, GPs can be defined to support point-to-point, pointto-multipoint or multipoint-to-multipoint connections between EPs.

The GP represents the connections between the same or different layers in a common way. In a top-down perspective, an end-to-end (E2E) GP (representing the overall path used in the connection) may for example be mapped at the physical level to a wireless channel (e.g. WiFi, WiMAX) or to a wavelength at an optical fiber (WDM). For example, considering a communication in a university campus, we can figure out the need for establishing a GP between two processes, each in a different terminal. At network level we can think of a single GP for the information dissemination mapping into a concatenation of several smaller GPs, at data link level, which at last are adequately mapped into physical channels (see Fig 1).

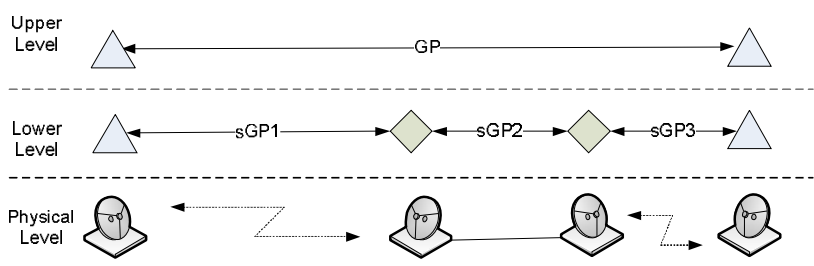

Fig. 1. Different GP levels mapping

Besides the ability to map and negotiate link properties, each GP is expected to efficiently react under unpredictable network dynamic events, so that the continuity of on-going transmissions with required QoS guarantees can be ensured. Examples of network dynamic events that endanger such transmissions are the radio conditions deterioration, load evolution and interface connectivity variations. In this sense, selforganization and resilience support is crucial, which must take into account performance issues in terms of scalability and optimized operations. 
It is expected that the GP abstraction should deal in an efficient way with multiplicity of connectivity interfaces, from the same or different technology, in a simultaneous (multihoming) or sequential manner (handover). In order to assure mobility is handled efficiently, each GP should "react" to the various scenarios characteristics, by providing the necessary API to dynamically add and remove EP's.

The GPs are created and managed in a network block called compartment. Each compartment is associated to a specific administrative domain and a particular technology support, defined at any level. This implies the existence of (e.g.) IP, Ethernet and TCP compartments, at different levels. Each GP branch will be the collected contribution of several sub-GPs branches linked within all the heterogeneous compartments that the information needs to travel through to reach the sink(s). In Fig 2, we illustrate this GP concatenation process in different compartments. The vertical compartments are called Node Compartments, and represent the processing system, or node.

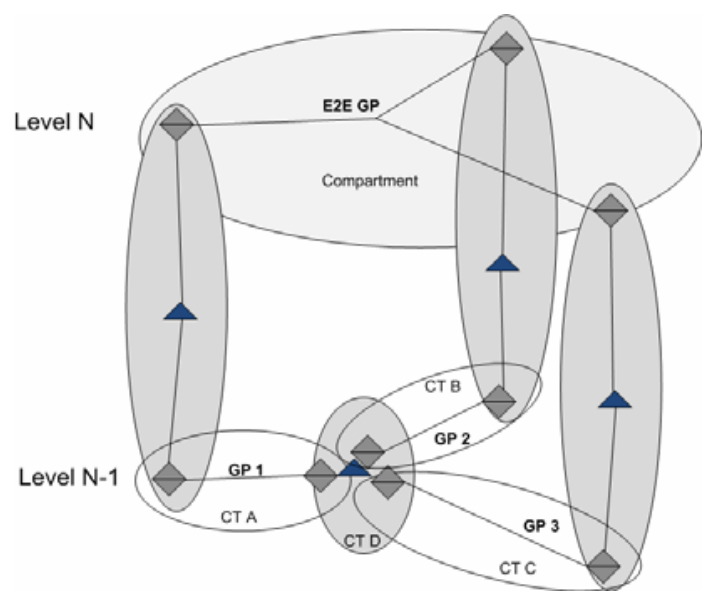

Fig. 2. GP existence within different levels

Mediation Points (MPs, triangles in blue) have the main purpose of interfacing different connections, by interconnecting EPs within the same CT, providing functions of buffering, multiplexing, transcoding and bit rate adaptability, assuring the information flow between distinct connections / GPs. For example, when a terminal moves out of range of the old base station, a branch of the GP could be torn down as the terminal no longer can communicate with the old base station - in that case the existence of mediation points in the base station seems like a valuable solution. To conclude, the MP has functionalities to "fork" GPs, that is, to discover and to set up alternative routing solutions to the same endpoint (e.g. multi-path routing mechanism is a possible mechanism for user mobility).

\subsection{The GPMR Concept}

In order to fulfill the end-to-end path requirements, such as connectivity (supported by multihoming, either with multiple interfaces or technologies), or the holy grail of 
mobility, seamless handover, the GP architecture must provide efficient means for control and support. As the information derived from the creation of each GP needs to be acted upon, a way is needed for collecting and controlling this information. We propose the introduction of a record for storing relevant characteristics from each of the GPs within its local compartment - the GPMR - and another one for storing the E2E (across all compartments) GP information - the Master Record (MR) a innovative solution relatively to nowadays networks that provides a unified framework about the communication path. As an abstraction, the GP depends on proper control, and as the element that stores all the information about the GP, GPMR can provide such feature.

The first issue to be tackled is the question "Where to locate the GPMR?" Several approaches were studied in our research. For the sake of brevity, we present the pros and cons of some alternate proposals considered for the GP record location (Table 1).

Table 1. GPMR Location Alternate Proposals

\begin{tabular}{lll}
\hline Location & Advantages & Disadvantages \\
\hline At each Entity & $\begin{array}{l}\text { Record is kept out of the network } \\
\text { core }\end{array}$ & $\begin{array}{l}\text { Entity heterogeneity compromises } \\
\text { implementation simplification }\end{array}$ \\
At both EP's & Adds resilience to the network & $\begin{array}{l}\text { Redundancy / Information } \\
\text { replication/synchronization adds } \\
\text { overhead }\end{array}$ \\
At the Source & $\begin{array}{l}\text { Process simplification as only one } \\
\text { element is responsible for each GP } \\
\text { record; } \\
\text { Source doesn't need to be concerned } \\
\text { about network topology changes }\end{array}$ & $\begin{array}{l}\text { EP needs to maintain a record; } \\
\text { Mobility makes tracing of GP status } \\
\text { difficult / impossible. }\end{array}$ \\
\hline
\end{tabular}

After evaluating aspects such as centralization and added value to path management, the best option seems to be placing the GPMR at each CT that the GP traverses, which results in multiple GPMRs "tracking" the same higher level GP. The advantages for such a solution include:

i. The decentralization of GP functionalities;

ii. Optimization in GP look-up operations;

iii. Technology and communication type independent (any type of GP can be described this way);

iv. A QoS aware element which allows relevant entities - Entities, GP Factory, In-Network Management (INM), etc - to trigger necessary routing / GP reconfiguration decisions;

v. Flexible solution that can be optimized for different mobility cases by simply varying the mobility indirections.

As for the disadvantages:

i. Considering very dynamic GPs: future studies must analyze scalability; how big can a CT be and how would the number of updates increase with it?

ii. From an end-to-end point of view, a MR is the result of the concatenation of various GPMRs, implying a large amount of information exchange. 
Fig 3 shows a possible relationship between MR and the different GPMRs. A, B and $\mathrm{C}$ correspond to different node CTs, and each of the records is able to communicate with other records, as long as they're responsible for a common higher level GP. Additionally, a GPMR may talk to more than on MR, e.g., when more than one higher level GP are simultaneously using a lower level GP.

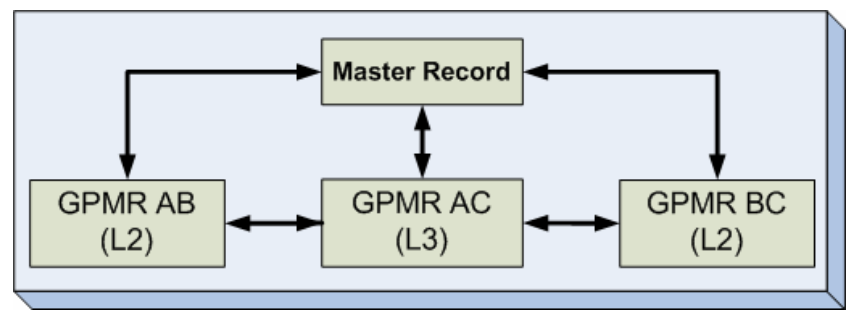

Fig. 3. MR Information Hierarchy

Fig 4 depicts the GPMR within a CT and the relationship with other functionalities. The GPMR is being defined to provide support for GP operations related to the GP factory, namely GP creation and destruction, and for later usage by mechanisms such as QoS-aware routing, traffic engineering, resilience, and so on. For instance, network management and control blocks should supply GPMR with up-to-date QoS-related information as a result of their network management and resource control operations. Available network control schemes must update QoS capabilities of a GP after setting up a bandwidth reservation along it, or periodically via soft-state operations. The same idea is also addressed for Traffic Engineering support.

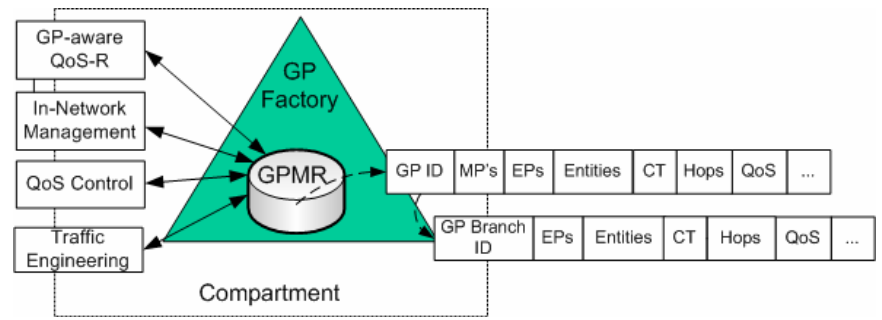

Fig. 4. GPMR Functionalities

Major issues about the GPMR and subject for future work include determining the amount of information that should be traded between GPMRs of different levels, a key aspect that must be well defined to better provide cross-layer optimization. A possible solution would be the module definition inside MR. Another problem is the GP architectural elements namespaces definitions, e.g. GP, EP. In future work, an analysis and enhancement of this solution by the introduction of Media Independent Handover (MIH) mechanisms is also intended. The IEEE 802.12 MIH standard [12] provides media independent mechanisms that aid and optimize the handover process, using commands and events to obtain information about different access technologies, enabling MIH-enhanced management entities to better select handover candidates. 
Although these mechanisms are more oriented towards handover operation, information is itself an important part in the GP global architecture. MIH mechanisms can be introduced as the providers of such information (either dynamic through the Media Independent Event Service) or static (Media Independent Information Service) enabling GP Factories to establish paths based on optimum choice conditions (i.e., optimize route by choosing nodes with low load and that better support a specific kind of traffic such as VoIP, or updating a path due to foreseen out-of-range from a terminal's current signal strength analysis). Also relevant is what kind of information objects should be used, because MIH focuses on handovers while GP also involves all the necessary steps for data communication, such as routing and session establishment.

\section{Mobility and Generic Paths}

The work around the definition of Future Internet should take special concern on mobility. The typical network user will roam between several different technologies while moving, and will need transparent, fast and accurate handover support.

\subsection{Mobility Types}

Supporting wide scale mobility raises new issues. Current IP network architectures do not provide satisfactory solutions (see e.g. [3]) but an inefficient handling of mobility via add-on mechanisms. Some of the main Internet shortcomings include the lack of support for multi-homing, fast handover, simultaneous multi-access, and nonintermittent connectivity in wireless networks. Those important requirements work as core drivers to new designs for mobility support in the Generic Path architecture.

As a starting point in our work, we distinguished the different types of mobility: user mobility, session mobility and network mobility.

User mobility implies the change of the point of attachment that best serves the user by the network, assuming the simultaneous movement of the Entity residing in the terminal. Within this approach we can also state that the corresponding moving end-point will need to change, in order to build a GP that better fits the new geographical position of the device. There is also the case where there is no movement of the user, but for guaranteeing the QoS levels the user may detach from the current access point and connect to another.

Session mobility occurs when the user plans to move the service between terminals. This takes places when, e.g., a user is participating in a video-conference, and as soon as it gets to his office plans to migrate it to the desktop computer. In our architectural approach this means that the end point and the Entity must be moved from one terminal to another terminal. The transference of the session can be triggered by the user or by the network. In this process, some similar steps to the previous approach should be taken. The GP established for the video-conference should somehow be redirected to the desktop, and MP functionalities will aid in traffic redirection and video transcoding.

As for network mobility, we can think of a scenario where a network is established in a vehicle, implying constant mobility. In such case, the GPs defined between the network nodes should be more or less static, except the ones connecting the vehicle to the outside network. 


\subsection{Mobility Taxonomy}

In order to provide the desirable mobility in the future Internet, several scenarios have been conceptually studied. As previously described the GP will be responsible for the flow of the information between the communications entities, so, as long as the endpoints have some sort of mobility, it will be necessary to develop a strategy for the handover mechanism and to maintain the communication with the desirable QoS parameters. Four different scenarios aiming to fulfill the requirements have been identified, with potential realizations in different CTs.

\section{Dynamic GP Modification}

The first approach, Dynamic GP Modification, is based in the constant GP updating each time that any kind of mobility occurs, fitting the overall concept of a moving GP. It can be stated that the GP becomes a lively entity, constantly being created, destroyed and modified in association to the physical realization. Such a GP vision grants that all GPs are fully flexible and dynamic in terms of behavior.

In the example of Fig 5, EP 1 communicates with EP 2 by means of an intermediary MP, for example due to physical distance. The trajectory that EP 1 did allowed it to become closer to EP 2, which in terms of GP modification, possibly by soft handover represented the following: i) creation of GP 3 and respective introduction into the CT GPMR, in an entry parallel to GP $1+$ GP 2, as it represents a E2E GP with common EPs; ii) sequential destruction of GP 1 and GP 2, and removal from GPMR. Regarding routing processes, as communication is done directly between the EPs, no routing / forwarding is now needed as before through the intermediary MP. Nevertheless, special concerns should be taken when adopting this method, due to the inherent delay in the creation / destruction of each new GP branch: problems associated with the synchronization and replication at the endpoints should be taken into account.

Concluding, this scenario involves fast and accurate creation and destruction of GP branches (e.g. GPs at a given CT), translating into a constant update of the GPMR, mostly at the data link and network levels, and in complex manageability when considering high mobility networks. This solution provides a simple and uniform scheme independently of the type of communications established, and could be optimally used in small or low mobility, for example in backbone and at the core of the network.

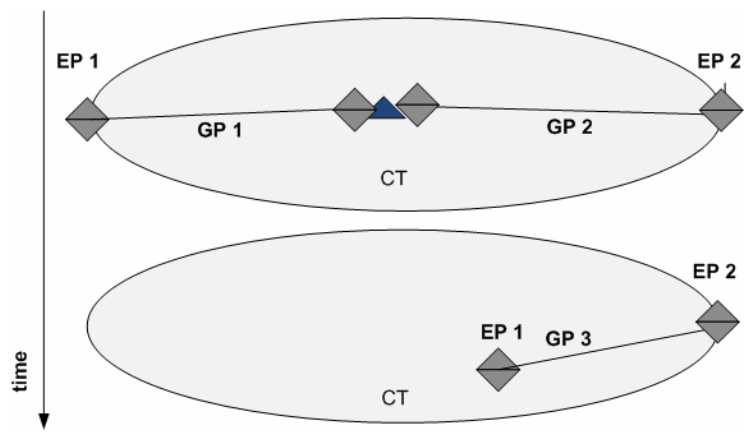

Fig. 5. Dynamic GP Modification Representation 


\section{Continuous Binding Scheme}

The second proposed solution is the Continuous Binding Strategy, where each GP is composed of two parts, each of them characterized by the associated EP type. The use of different kinds of EPs by a GP was first presented in Anchorless Mobility approach [14]. We can state that this scheme is centered on the EP binding function. In its most basic design, a connectivity EP detaches from a locator EP and binds to the next available locator EP. GP is physically reconfigured, maintaining the communication between entities. This implies that the GP is actually built from two parts, one between locators EPs (or MPs), and the other between the connectivity and logical EPs, as shown in Fig 6.

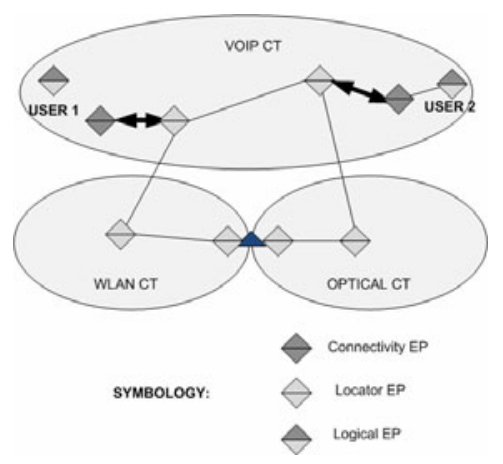

Fig. 6. Continuous binding scheme representation

\section{Static Multiconnection P2MP}

The third proposed solution is the Static Multiconnection P2MP GP, which assures strong mobility coverage without the need for a binding function. Such mobility solution is targeting wireless technologies, and is possible mostly at the physical level, as the main decision factor is the physical coverage area, measured in terms of transmission power. We propose the creation of a point-to-multipoint GP that assures persistent coverage of the user whatever its current geographic position is (Fig 7). This level of connectivity is supported not only by its coverage cell, but also by the neighbor cells.

As advantages, we can refer the reduced amount of mechanisms needed for small mobility scenarios, avoiding the delay inherent to the constant creation, destruction or changing in the GP that assures the communication. That is also a consequence in terms of low GPMR amount of updating. We also avoid the disadvantages of supporting binding functions. On the other side, it brings a traffic increment because of the need for broadcasting, as the same data flow is sent to the user and to all the other network points on its vicinity.

As solutions that are included in this scheme, we can immediately think of a swarm scenario, and other P2MP or MP2MP schemes, as the newly proposed Concurrent Multipath Transfer [14]. 


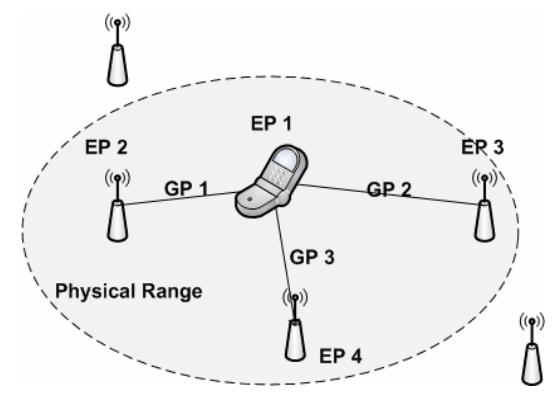

Fig. 7. Static multiconnection P2MP example

\section{EP as an Anchor}

As a last solution, we present the EP as a simple Anchor. In this approach we use the concept of anchoring, as the EP can be considered as an anchor that serves a particular geographical area. So as long as the user moves in between this area, the anchor serves as data flow supplier to all the network access points.

In Fig 8, EP 1 is communicating with both EP 2 and EP 3. As can be seen, all traffic towards EP 1 is handled by the Anchor EP, as long as EP 1 is within the anchor range. In the moment that EP 1 leaves this range, a new Anchor EP is obtained, either by EP 1 or by the actual anchor EP request.

This last solution provides simplicity and also avoids the concern about the mobility of the GP restrained to the mobility of the user. However, in this scheme it is necessary to gather information about macroscopic localization of the user, as well as controlling the data flow as soon as the user leaves the anchor coverage zone. In terms of GPMR, the level of updating varies depending on the level in which the GPMR applies; the level of updating is null when the mobility is within the anchor range, avoiding considerable overhead. Solutions that can be integrated in this scheme are the already proposed hierarchical mobility schemes and also an innovative scheme, Dynamic Mobility Anchoring [14] proposed within 4WARD project.

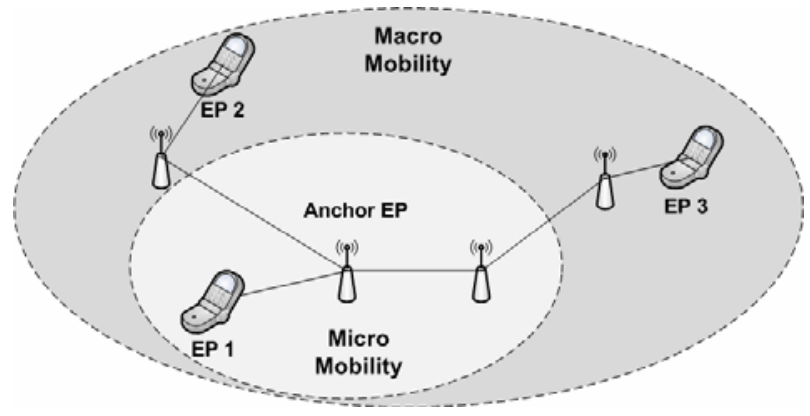

Fig. 8. EP as an anchor example 


\subsection{Paradigms Comparison}

This section provides a resume of each of the mobility schemes in terms of some of the most relevant properties, such as flexibility or scalability. Naturally, these models will be associated with the specific technology realization, and its inherent constrains.

Table 2. Comparison mobility models for GP architecture

\begin{tabular}{lllll}
\hline & $\begin{array}{l}\text { Dynamic GP } \\
\text { Modification }\end{array}$ & $\begin{array}{l}\text { Continuous } \\
\text { Binding Scheme }\end{array}$ & $\begin{array}{l}\text { Static } \\
\text { Multiconnection } \\
\text { P2MP }\end{array}$ & $\begin{array}{l}\text { EP as an } \\
\text { Anchor }\end{array}$ \\
\hline $\begin{array}{l}\text { Flexibility } \\
\begin{array}{l}\text { Delay associated to } \\
\text { operations }\end{array}\end{array}$ & $\begin{array}{l}\text { High } \\
\text { Depends on level } \\
\text { of mobility }\end{array}$ & $\begin{array}{l}\text { High } \\
\text { Average }\end{array}$ & $\begin{array}{l}\text { Average } \\
\text { Low }\end{array}$ & High \\
Connectivity Strength & $\begin{array}{l}\text { Average } \\
\text { Average }\end{array}$ & $\begin{array}{l}\text { Average } \\
\text { Average }\end{array}$ & $\begin{array}{l}\text { Large } \\
\text { High }\end{array}$ & $\begin{array}{l}\text { Large } \\
\text { Average }\end{array}$
\end{tabular}

\section{Conclusions and Future Work}

This paper started describing the clean-slate approach brought by the Generic Path concept for the future Internet. We have introduced in a brief manner the architectural elements related to the GP approach and its importance in mobility. The GPMR concept was originally proposed as a solution for keeping a record about the state of a GP, providing a powerful tool for being used in mobility scenarios. Regarding the usage of MIH mechanisms it is still unclear at this point in time the best way to integrate MIH into our GP architecture, due different objectives of both approaches and due to the GP as a still evolving solution. We are currently evaluating how to best apply 802.21 functionality to GP boxes and how to adapt MIH-alike behavior into GP concepts.

We presented several models for the types of mobility for Future Internet under the scope of the GP architecture, obtaining the most relevant solutions, with each of them comprising a broad range of mobility scenarios and mapping to more concrete cases.

Our current research is aiming to evaluate and compare those different approaches with real technologies and how they could coexist, in order to provide the best approach for dealing with the mobility issues for the future Internet definition in the 4WARD Project.

\section{Acknowledgments}

We would like to thank our colleagues in the 4WARD Work Package 5, aptly titled Generic Paths, for fruitful discussions, in particular to our research group at ITAveiro. Special thanks go to Eng.. Daniel Corujo, who shared with us an interesting view on a possible interaction between MIH and GP architecture.

This work has been carried out in the framework of the IST 7th Framework Programme Integrated Project 4WARD, which is partially funded by the Commission of the European Union. The views expressed in this paper are solely those of the authors 
and do not necessarily represent the views of their employers, the 4WARD project, or the Commission of the European Union.

\section{References}

[1] The FP7 4WARD Project, http: / /www . 4ward-project. eu /

[2] Johnson, D., Perkins, C., Arkko, J.: IP Mobility Support in IPv6, RFC3775, IETF (June 2004)

[3] Soliman, H., et al.: Hierarchical Mobile IPv6 Mobility Management (HMIPv6). rfc4140.txt, IETF (August 2005)

[4] Floyd, S., Kohler, E.: Profile for Datagram Congestion Control Protocol (DCCP) Congestion Control ID 2: TCP-like Congestion Control. RFC 4341, Internet Engineering Task Force (March 2006)

[5] Stewart, R., Metz, C.: SCTP: new transport protocol for TCP/IP, Internet Computing. IEEE, Los Alamitos (2001)

[6] Siekkinen, M., Goebel, V., Plagemann, T., Skevik, K.A., Banfield, M., Brusic, I.: Beyond the Future Internet-Requirements of Autonomic Networking Architectures to Address Long Term Future Networking Challenges. In: 11th IEEE International Workshop on FTDCS (March 2007)

[7] Gavras, A., Karila, A., Fdida, S., May, M., Potts, M.: Hierarchical Broadcasting in the Future Mobile Internet 37(3) (July 2007)

[8] Wong, W., Villaca, R., de Paula, L.B., Pasquini, R., Verdi, F.L., Magalhaes, M.F.: An Architecture for Mobility Support in a Next-Generation Internet. In: AINA 2008 (March 2008)

[9] Guillemin, F., Woesner, H.: Path Generalizations in a Functional Architecture. In: ICT, Mobile Summit 2008, Stockholm (2008)

[10] Guillemin, F.: Architecture of a Generic Path, 4WARD Deliverable D5.1 (2008)

[11] Söllner, M., et al.: Mobility Scenarios for the Future Internet: the 4WARD Approach. In: WPMC 2008 (2008)

[12] IEEE 802.21 Standard, Local and Metropolitan Area Networks - Part 21: Media Independent Handover Services (January 2009)

[13] Matos, A., Aguiar, R.L.: Mobility Aware Paths: The Identity Connection. In: WPMC 2008 (2008)

[14] Bertin, P., Aguiar, R.L., Folke, M., Schefczik, P., Zhang, X.: Paths to Mobility Support in Future Internet. In: ICT 2009 (2009) 